\title{
METAPLECTIC FORMULATION OF THE WIGNER TRANSFORM AND APPLICATIONS
}

\author{
NUNO COSTA DIAS, MAURICE A. DE GOSSON, AND JOÃO NUNO PRATA
}

\begin{abstract}
We show that the cross Wigner function can be written in the form $W(\psi, \phi)=\hat{S}(\psi \otimes \overline{\hat{\phi}})$ where $\hat{\phi}$ is the Fourier transform of $\phi$ and $\hat{S}$ is a metaplectic operator that projects onto a linear symplectomorphism $S$ consisting of a rotation along an ellipse in phase space (or in the time-frequency space). This formulation can be extended to generic Weyl symbols and yields an interesting fractional generalization of the Weyl-Wigner formalism. It also provides a suitable approach to study the Bopp phase space representation of quantum mechanics, familiar from deformation quantization. Using the "metaplectic formulation" of the Wigner transform we construct a complete set of intertwiners relating the Weyl and the Bopp pseudo-differential operators. This is an important result that allows us to prove the spectral and dynamical equivalence of the Schrödinger and the Bopp representations of quantum mechanics.
\end{abstract}

MSC [2000]: Primary 35S99, 35P05; Secondary 35S05, 45A75

\section{INTRODUCTION}

It is well known that the cross Wigner function

$$
W(\psi, \phi)(x, p)=\frac{1}{(2 \pi)^{n}} \int e^{i p \cdot \xi_{p}} \psi\left(x-\frac{\xi_{p}}{2}\right) \bar{\phi}\left(x+\frac{\xi_{p}}{2}\right) d \xi_{p}
$$

defined for arbitrary $\phi, \psi \in L^{2}\left(\mathbb{R}^{n}\right)$, satisfies the Moyal identity

$$
\|W(\psi, \phi)\|_{L^{2}\left(\mathbb{R}^{2 n}\right)}=\frac{1}{(2 \pi)^{n / 2}}\|\phi\|_{L^{2}\left(\mathbb{R}^{n}\right)}\|\psi\|_{L^{2}\left(\mathbb{R}^{n}\right)} .
$$

Hence, for $\|\phi\|_{L^{2}\left(\mathbb{R}^{n}\right)}=1$, the mapping

$$
W_{\phi}: L^{2}\left(\mathbb{R}^{n}\right) \longrightarrow L^{2}\left(\mathbb{R}^{2 n}\right) ; \psi \longmapsto W_{\phi} \psi=(2 \pi)^{n / 2} W(\psi, \phi)
$$

called the windowed Wigner transform, is a non-surjective isometry $L^{2}\left(\mathbb{R}^{n}\right) \rightarrow$ $L^{2}\left(\mathbb{R}^{2 n}\right)$.

In this paper we will further show that $W_{\phi}$ can be written in the form

$$
W_{\phi} \psi=\hat{S}(\psi \otimes \overline{\hat{\phi}})
$$

where $\hat{S}=e^{-i \theta_{0} \hat{H}}$ is the unitary operator generated by the self-adjoint Hamiltonian

$$
\hat{H}=2 \hat{\xi}_{x} \cdot \hat{\xi}_{p}-\left(\hat{x} \cdot \hat{\xi}_{x}\right)_{+}-\left(\hat{p} \cdot \hat{\xi}_{p}\right)_{+}+4 \hat{x} \cdot \hat{p}
$$


and $\theta_{0}$ is a suitable value of the scalar parameter. In (44) the subscript + denotes symmetrization and

$$
\hat{x}=x \cdot \quad, \quad \hat{\xi}_{x}=-i \partial_{x} \quad \text { and } \quad \hat{p}=p \cdot \quad, \quad \hat{\xi}_{p}=-i \partial_{p}
$$

are the fundamental operators in the phase space Schrödinger representation of the Heisenberg algebra (thus acting on functions $\Psi(x, p) \in D(\hat{H}) \subset$ $\left.L^{2}\left(\mathbb{R}^{n} \times \mathbb{R}^{n}\right)\right)[9$.

Since $\hat{H}$ is quadratic, the transformation $\hat{S}$ is metaplectic [12, 14, 16]. Its projection onto the group of linear symplectic transformations $\operatorname{Sp}(4 n)$ of $\mathbb{R}^{2 n} \times \mathbb{R}^{2 n}$ is a symplectomorphism generated by the classical Hamiltonian

$$
H=2 \xi_{x} \cdot \xi_{p}-x \cdot \xi_{x}-p \cdot \xi_{p}+4 x \cdot p
$$

and consists of a rotation along an ellipse in the phase space.

The "metaplectic formulation" of the Wigner function can be extended to generic Weyl operators. The resulting formalism yields new calculation tools for the Weyl-Wigner calculus and displays several interesting applications. We will use it to study a new topic and to revisit an old subject.

The new topic is a fractional generalization of the Weyl-Wigner formalism. Using the metaplectic approach we construct a fractional version of both the Wigner function and the Weyl symbols. Moreover, we generalize several of the key properties of the standard Weyl-Wigner framework to the fractional case. These include the regularity properties, inversion and kernel formulas and the composition formula [16, 25]. We also study the main features of the associated fractional quantization.

The old topic is the Bopp representation of quantum mechanics [4, 5, 9, 16]. This is a phase space operator representation that was recently used [9, 17] to prove precise spectral results for the deformation quantization of Bayen et al [1, 2, 10, 11]. A similar formulation was used in [15, 18] to study the spectral properties of generalized Landau operators [19], and in [7, 8. to address the spectral problem in noncommutative quantum mechanics. In another recent paper [3], a generalization of the Bopp representation was used to address the problem of determining a consistent formulation of coupled classical quantum dynamics [6, 23. The results of [3] suggest that the phase space representations are more suitable to address this problem than the standard configurational space ones. Here, we will focus on the mathematical formalism and use the metaplectic representation of the Wigner transform to determine a complete family of non-surjective isometries that intertwines the Weyl and the Bopp pseudo-differential operators. A straightforward consequence of this result is that the Schrödinger and the Bopp representations of quantum mechanics (although not unitarily related) display equivalent spectral and dynamical properties.

This paper is organized as follows: in the next section we present the metaplectic formulation of the Wigner function (section 2.2) and Weyl symbols (section 2.3). In section 3 we define and study the fractional generalization of the Weyl calculus. In section 4 we use the metaplectic formulation to 
study the spectral and dynamical properties of the Bopp representation of quantum mechanics.

Notation 1. A generic point in the double phase space $\mathbb{R}^{4 n}=\mathbb{R}^{2 n} \oplus \mathbb{R}^{2 n}$ is denoted by $z=\left(x, p, \xi_{x}, \xi_{p}\right)$ where $\left(x, \xi_{x}\right) \in \mathbb{R}^{2 n}$ and $\left(p, \xi_{p}\right) \in \mathbb{R}^{2 n}$ are canonical conjugate pairs. The standard sympletic form on $\mathbb{R}^{4 n}$ is $\sigma\left(z, z^{\prime}\right)=$ $\xi_{x} \cdot x^{\prime}+\xi_{p} \cdot p^{\prime}-\xi_{x}^{\prime} \cdot x-\xi_{p}^{\prime} \cdot p$ and the corresponding sympletic group is $S p(4 n)$. The metaplectic group $M p(4 n)$ is the unitary representation of the double cover of $S p(4 n)$.

Notation 2. The norm and the inner product in $L^{2}\left(\mathbb{R}^{n}\right)$ are denoted by $\|\cdot\|_{L^{2}\left(\mathbb{R}^{n}\right)}$ and $(\cdot, \cdot)_{L^{2}\left(\mathbb{R}^{n}\right)}$, or just by $\|\cdot\|$ and $(\cdot, \cdot)$ whenever the dimension of the functions' domain is clear from the context. The distributional bracket is $\langle\cdot, \cdot\rangle$.

For $f: \mathbb{R}^{n} \longrightarrow \mathbb{C}$ and $g: \mathbb{R}^{n} \longrightarrow \mathbb{C}, f \otimes g$ denotes the tensor product function

$$
f \otimes g: \mathbb{R}^{n} \times \mathbb{R}^{n} \longrightarrow \mathbb{C} ;(x, y) \longmapsto f \otimes g(x, y)=f(x) g(y)
$$

Operators are denoted by roman letters with a hat (the exceptions are $\hat{\xi}_{x}$ and $\hat{\xi}_{p}$ ). The hat also denotes the Fourier transform (Notation 3) but it should always be clear from the context what it refers to.

Notation 3. The partial Fourier and inverse Fourier transforms are written:

$$
\hat{\Psi}\left(x, \xi_{p}\right)=\mathcal{F}_{p \rightarrow \xi_{p}}[\Psi(x, p)]=\frac{1}{(2 \pi)^{n / 2}} \int_{\mathbb{R}^{n}} e^{-i \xi_{p} \cdot p} \Psi(x, p) d p
$$

and

$$
\check{\Psi}(x, p)=\mathcal{F}_{\xi_{p} \rightarrow p}^{-1}\left[\Psi\left(x, \xi_{p}\right)\right]=\frac{1}{(2 \pi)^{n / 2}} \int_{\mathbb{R}^{n}} e^{i \xi_{p} \cdot p} \Psi\left(x, \xi_{p}\right) d \xi_{p}
$$

and are defined as unitary operators in $L^{2}\left(\mathbb{R}^{2 n}\right)$ (for the Fourier transform in $L^{2}\left(\mathbb{R}^{n}\right)$ we use exactly the same notation). As usual they can be extended by duality to $\mathcal{S}^{\prime}\left(\mathbb{R}^{2 n}\right)$. Notice that when we write $\mathcal{F}_{p \rightarrow \xi_{p}}[\Psi(x, p)],(x, p)$ is the argument of the original function and the result is a function of $\left(x, \xi_{p}\right)$. On the other hand, if we write $\mathcal{F}_{p \rightarrow \xi_{p}} \Psi\left(x, \xi_{p}\right)$ then $\left(x, \xi_{p}\right)$ is already the argument of $\mathcal{F}_{p \rightarrow \xi_{p}} \Psi$.

\section{Metaplectic formulation of the Wigner transform}

In this section we prove our main result. It states that $W(\psi, \phi)=$ $(2 \pi)^{-n / 2} \hat{S}(\psi \otimes \overline{\hat{\phi}})$ where $\hat{S}=e^{-i \theta_{0} \hat{H}}$ is the unitary operator generated by the Hamiltonian (44) for $\theta_{0}=\frac{\sqrt{7}}{7} \arccos \frac{3}{4}$. We will also extend this formulation to generic Weyl symbols. 
2.1. The classical symplectomorphism. The unitary transformation $\hat{S}$ is generated by the quadratic Hamiltonian (4) and thus belongs to the metaplectic group $\operatorname{Mp}\left(\mathbb{R}^{4 n}\right)$ [12, 13, 16, 21. Its projection onto the group of symplectic transformations of $\left(\mathbb{R}^{4 n}, \sigma\right)$ is a symplectomorphism $S$ belonging to the one-parameter group of symplectomorphisms $s(\theta)$ generated by the classical Hamiltonian (6). In order to prepare our main results, we now determine the explicit form of $s(\theta)$ and $S$.

Theorem 4. Let $s: \mathbb{R} \times \mathbb{R}^{4 n} \longrightarrow \mathbb{R}^{4 n}$ be the one-parameter group of symplectic transformations generated by the classical Hamiltonian

$$
H=2 \xi_{x} \cdot \xi_{p}-x \cdot \xi_{x}-p \cdot \xi_{p}+4 x \cdot p .
$$

For $\theta_{0}=\frac{\sqrt{7}}{7} \arccos \frac{3}{4}$ the symplectomorphism $S=s\left(\theta_{0}\right)$ is explicitly

$$
S: \mathbb{R}^{4 n} \longrightarrow \mathbb{R}^{4 n}, \quad\left\{\begin{array}{l}
x \longmapsto x / 2+\xi_{p} / 2 \\
\xi_{x} \longmapsto \xi_{x}-p \\
p \longmapsto p / 2+\xi_{x} / 2 \\
\xi_{p} \longmapsto \xi_{p}-x
\end{array} .\right.
$$

Moreover, $S$ is the natural projection of $\hat{S}=e^{-i \theta_{0} \hat{H}} \in M p(4 n)$ onto $S p(4 n)$.

Proof. The map $s(\theta)=\left(x(\theta), p(\theta), \xi_{x}(\theta), \xi_{p}(\theta)\right)$ is defined by the Hamilton equations

$$
\left\{\begin{array}{l}
\dot{x}=\frac{\partial H}{\partial \xi_{x}}=2 \xi_{p}-x \\
\dot{p}=\frac{\partial H}{\partial \xi_{p}}=2 \xi_{x}-p \\
\dot{\xi}_{x}=-\frac{\partial H}{\partial x}=\xi_{x}-4 p \\
\dot{\xi}_{p}=-\frac{\partial H}{\partial p}=\xi_{p}-4 x
\end{array}\right.
$$

which decouple into two systems

$$
\left\{\begin{array}{l}
\dot{x}=\frac{\partial H}{\partial \xi_{x}}=2 \xi_{p}-x \\
\dot{\xi}_{p}=-\frac{\partial H}{\partial p}=\xi_{p}-4 x
\end{array} \quad, \quad\left\{\begin{array}{l}
\dot{p}=\frac{\partial H}{\partial \xi_{p}}=2 \xi_{x}-p \\
\dot{\xi}_{x}=-\frac{\partial H}{\partial x}=\xi_{x}-4 p
\end{array}\right.\right.
$$

with solutions $(k=\sqrt{7})$

$$
\left\{\begin{array}{l}
x(\theta)=x(0)\left[\cos k \theta-k^{-1} \sin k \theta\right]+2 k^{-1} \xi_{p}(0) \sin k \theta \\
\xi_{p}(\theta)=\xi_{p}(0)\left[\cos k \theta+k^{-1} \sin k \theta\right]-4 k^{-1} x(0) \sin k \theta
\end{array}\right.
$$

and

$$
\left\{\begin{array}{l}
p(\theta)=p(0)\left[\cos k \theta-k^{-1} \sin k \theta\right]+2 k^{-1} \xi_{x}(0) \sin k \theta \\
\xi_{x}(\theta)=\xi_{x}(0)\left[\cos k \theta+k^{-1} \sin k \theta\right]-4 k^{-1} p(0) \sin k \theta
\end{array} .\right.
$$

Hence, $s(\theta)$ is given explicitly by the equations (819). For

$$
\theta=k^{-1} \arccos \frac{3}{4}=k^{-1} \arcsin \frac{k}{4}
$$

these equations yield the transformation $S$ exactly. 
Since $H$ is the Weyl symbol of $\hat{H}$ (given by (4)), the symplectomorphisms $s(\theta)$ are the projections onto $\operatorname{Sp}(4 n)$ of the metaplectic operators $\hat{U}(\theta)=$ $e^{-i \theta \hat{H}}$. So, in particular, $S=s\left(\theta_{0}\right)$ is the projection of $\hat{S}=\hat{U}\left(\theta_{0}\right)$.

Remark 5. The family of transformations $s(\theta)$ is periodic. We have $s(\theta+$ $\left.\frac{2 \pi}{k}\right)=s(\theta)$ for all $\theta \in \mathbb{R}$. The orbits generated by $s$ belong to the following level surfaces

$$
\left\{\begin{array}{l}
2 x^{2}+\xi_{p}^{2}-x \xi_{p}=\text { Const } \\
2 p^{2}+\xi_{x}^{2}-p \xi_{x}=\text { Const }^{\prime}
\end{array}\right.
$$

and consist of a rotation along two ellipses in the $x, \xi_{p}$ and $p, \xi_{x}$ planes of the phase space.

2.2. The main result. Let $\hat{T}(\theta)$ be the one-parameter (periodic) group of unitary transformations defined by

$$
\hat{T}(\theta): L^{2}\left(\mathbb{R}^{2 n}\right) \longrightarrow L^{2}\left(\mathbb{R}^{2 n}\right) ; \Phi\left(x, \xi_{p}\right) \longmapsto \hat{T}(\theta) \Phi\left(x, \xi_{p}\right)=\Phi\left(x(-\theta), \xi_{p}(-\theta)\right)
$$

where $x(-\theta)$ and $\xi_{p}(-\theta)$ are given by (8) . Let us also define $\hat{T}=\hat{T}\left(\theta_{0}\right)$.

We start by proving the following

Lemma 6. The one-parameter unitary evolution group $\hat{U}(\theta)=e^{-i \theta \hat{H}}$ is given explicitly by

$$
\begin{gathered}
\hat{U}(\theta): L^{2}\left(\mathbb{R}^{2 n}\right) \longrightarrow L^{2}\left(\mathbb{R}^{2 n}\right) \\
\hat{U}(\theta) \Psi(x, p)=\mathcal{F}_{\xi_{p} \rightarrow p}^{-1} \hat{T}(\theta) \mathcal{F}_{p \rightarrow \xi_{p}}[\Psi(x, p)]
\end{gathered}
$$

Proof. Since $\hat{H}$ is self-adjoint and $\mathcal{S}\left(\mathbb{R}^{2 n}\right) \subset D(\hat{H})$, the initial value problem

$$
i \frac{\partial \Psi}{\partial \theta}=\hat{H} \Psi \quad, \quad \Psi(\cdot, 0)=\Psi_{0}(\cdot) \in \mathcal{S}\left(\mathbb{R}^{2 n}\right)
$$

has the unique solution

$$
\Psi(x, p, \theta)=e^{-i \theta \hat{H}} \Psi_{0}(x, p)
$$

where $e^{-i \theta \hat{H}}: L^{2}\left(\mathbb{R}^{2 n}\right) \longrightarrow L^{2}\left(\mathbb{R}^{2 n}\right)$ is the strongly continuous unitary evolution group generated by $\hat{H}$ (see, for instance [Chapter 5, 22]]). Our task is then to determine the solution of (13) explicitly.

The Hamiltonian operator $\hat{H}$ (4) can be re-written as

$$
\hat{H}=\mathcal{F}_{\xi_{p} \rightarrow p}^{-1}\left[-2 i \xi_{p} \cdot \partial_{x}+i x \cdot \partial_{x}-i \xi_{p} \cdot \partial_{\xi_{p}}+4 i x \cdot \partial_{\xi_{p}}\right] \mathcal{F}_{p \rightarrow \xi_{p}} .
$$

where the symmetric terms of (4) were calculated explicitly. Defining $\hat{\Psi}\left(x, \xi_{p}, \theta\right)=$ $\mathcal{F}_{p \rightarrow \xi_{p}}[\Psi(x, p, \theta)]$, we obtain from eq.(13)

$$
\begin{gathered}
i \frac{\partial \hat{\Psi}}{\partial \theta}=\left[-2 i \xi_{p} \cdot \partial_{x}+i x \cdot \partial_{x}-i \xi_{p} \cdot \partial_{\xi_{p}}+4 i x \cdot \partial_{\xi_{p}}\right] \hat{\Psi} \\
\hat{\Psi}(\cdot, 0)=\hat{\Psi}_{0}(\cdot) \in \mathcal{S}\left(\mathbb{R}^{2 n}\right) .
\end{gathered}
$$


The solution of this initial value problem is easily found to be:

$$
\hat{\Psi}\left(x, \xi_{p}, \theta\right)=\hat{\Psi}_{0}\left(x(-\theta), \xi_{p}(-\theta)\right)=\hat{T}(\theta) \hat{\Psi}_{0}\left(x, \xi_{p}\right) .
$$

Moreover, if $\hat{\Psi}_{0} \in \mathcal{S}\left(\mathbb{R}^{2 n}\right)$ then $\hat{\Psi}\left(x, \xi_{p}, \theta\right) \in \mathcal{S}\left(\mathbb{R}^{2 n}\right)$ for all $\theta$.

Consequently, the solution of eq.(13) is

$$
\Psi(x, p, \theta)=\mathcal{F}_{\xi_{p} \rightarrow p}^{-1}\left[\hat{\Psi}\left(x(-\theta), \xi_{p}(-\theta)\right)\right]=\mathcal{F}_{\xi_{p} \rightarrow p}^{-1} \hat{T}(\theta) \mathcal{F}_{p \rightarrow \xi_{p}}\left[\Psi_{0}(x, p)\right] .
$$

Let us then define

$$
\hat{U}(\theta): L^{2}\left(\mathbb{R}^{2 n}\right) \rightarrow L^{2}\left(\mathbb{R}^{2 n}\right) ; \quad \hat{U}(\theta) \Psi_{0}(x, p):=\mathcal{F}_{\xi_{p} \rightarrow p}^{-1} \hat{T}(\theta) \mathcal{F}_{p \rightarrow \xi_{p}}\left[\Psi_{0}(x, p)\right] .
$$

We conclude that $\hat{U}(\theta)$ is a linear and unitary (so continuous) operator and satisfies

$$
\left.\hat{U}(\theta)\right|_{\mathcal{S}\left(\mathbb{R}^{2 n}\right)}=\left.e^{-i \theta \hat{H}}\right|_{\mathcal{S}\left(\mathbb{R}^{2 n}\right)} .
$$

Since both operators are continuous (and $\mathcal{S}\left(\mathbb{R}^{2 n}\right)$ is dense in $L^{2}\left(\mathbb{R}^{2 n}\right)$ ) we also have in $L^{2}\left(\mathbb{R}^{2 n}\right)$,

$$
\hat{U}(\theta)=e^{-i \theta \hat{H}}
$$

which completes the proof.

It follows that

Theorem 7. Let $\hat{S}=\hat{U}\left(\theta_{0}\right)$ and $\theta_{0}=\frac{\sqrt{7}}{7} \arccos \left(\frac{3}{4}\right)$. For $\psi, \phi \in L^{2}\left(\mathbb{R}^{n}\right)$ we have

$$
W(\psi, \phi)=\frac{1}{(2 \pi)^{n / 2}} \hat{S}(\psi \otimes \overline{\hat{\phi}}) .
$$

Proof. We notice that

$$
\overline{\hat{\phi}}(p)=\overline{\mathcal{F}_{y \rightarrow p}[\phi(y)]}=\mathcal{F}_{y \rightarrow p}^{-1}[\bar{\phi}(y)]
$$

and so

$$
\psi \otimes \overline{\hat{\phi}}(x, p)=\mathcal{F}_{y \rightarrow p}^{-1}[\psi \otimes \bar{\phi}(x, y)] .
$$

We also notice from (8) and (11) that

$$
\hat{T} \Phi\left(x, \xi_{p}\right)=\Phi\left(x\left(-\theta_{0}\right), \xi_{p}\left(-\theta_{0}\right)\right)=\Phi\left(x-\xi_{p} / 2, x+\xi_{p} / 2\right) .
$$

Hence, we get

$$
\begin{aligned}
\hat{S}(\psi \otimes \overline{\hat{\phi}})(x, p) & =\mathcal{F}_{\xi_{p} \rightarrow p}^{-1} \hat{T} \mathcal{F}_{p \rightarrow \xi_{p}} \mathcal{F}_{y \rightarrow p}^{-1}[\psi \otimes \bar{\phi}(x, y)]=\mathcal{F}_{\xi_{p} \rightarrow p}^{-1}\left[\hat{T} \psi \otimes \bar{\phi}\left(x, \xi_{p}\right)\right] \\
& =\frac{1}{(2 \pi)^{n / 2}} \int_{\mathbb{R}^{n}} e^{i p \cdot \xi_{p}} \psi\left(x-\xi_{p} / 2\right) \bar{\phi}\left(x+\xi_{p} / 2\right) d \xi_{p}
\end{aligned}
$$

which is precisely $(2 \pi)^{n / 2} W(\psi, \phi)$. 
2.3. Metaplectic formulation of Weyl symbols. Let $\mathscr{L}\left(\mathcal{S}\left(\mathbb{R}^{n}\right), \mathcal{S}^{\prime}\left(\mathbb{R}^{n}\right)\right)$ be the space of linear and continuous operators of the form $\mathcal{S}\left(\mathbb{R}^{n}\right) \longrightarrow$ $\mathcal{S}^{\prime}\left(\mathbb{R}^{n}\right)$. The Schwartz kernel theorem states that all operators $\hat{a} \in \mathscr{L}\left(\mathcal{S}\left(\mathbb{R}^{n}\right), \mathcal{S}^{\prime}\left(\mathbb{R}^{n}\right)\right)$ admit a kernel representation

$$
\hat{a} \psi(x)=\left\langle K_{a}(x, \cdot), \psi(\cdot)\right\rangle
$$

where $K_{a} \in \mathcal{S}^{\prime}\left(\mathbb{R}^{n} \times \mathbb{R}^{n}\right)$. The Weyl symbol of $\hat{a}$ is defined by

$$
a(x, p)=\int_{\mathbb{R}^{n}} e^{-i p \cdot y} K_{a}\left(x+\frac{1}{2} y, x-\frac{1}{2} y\right) d y
$$

where the Fourier transform is taken in the distributional sense. We will denote by $\hat{a} \stackrel{\text { Weyl }}{\longleftrightarrow} a$ the one-to-one association between pseudo-differential operators (i.e. linear and continuous operators from $\mathcal{S}\left(\mathbb{R}^{n}\right)$ to $\mathcal{S}^{\prime}\left(\mathbb{R}^{n}\right)$ ) and Weyl symbols.

From (17) we immediately obtain for all $\phi \in \mathcal{S}\left(\mathbb{R}^{n}\right)$

$$
\langle\hat{a} \psi, \bar{\phi}\rangle=\left\langle K_{a}(x, y), \psi \otimes \bar{\phi}(y, x)\right\rangle
$$

and it is well known that this equation can be re-written in terms of the cross Wigner function and the Weyl symbol of $\hat{a}$, yielding the relation [16]

$$
\langle\hat{a} \psi, \bar{\phi}\rangle=\langle a, W(\psi, \phi)\rangle \text {. }
$$

Since the Wigner function $W(\psi, \phi)$ is (proportional to) the Weyl symbol of the operator with kernel $\psi \otimes \bar{\phi}$, we may suspect that a metaplectic formulation is also possible for generic Weyl symbols. This result is the content of the next theorem.

Theorem 8. Let $\hat{a}: \mathcal{S}\left(\mathbb{R}^{n}\right) \longrightarrow \mathcal{S}^{\prime}\left(\mathbb{R}^{n}\right)$ be a generic pseudo-differential operator, and let $K_{a} \in \mathcal{S}^{\prime}\left(\mathbb{R}^{n} \times \mathbb{R}^{n}\right)$ be the kernel of $\hat{a}$. Then the Weyl symbol $a$ of $\hat{a}$ is given by

$$
a(x, p)=(2 \pi)^{n / 2} \hat{S} \mathcal{F}_{y \rightarrow p}^{-1}\left[K_{a}(x, y)\right]
$$

where $\hat{S}=\hat{U}\left(\theta_{0}\right)$ is given by (12).

Proof. We note that for arbitrary $K_{a} \in \mathcal{S}^{\prime}\left(\mathbb{R}^{n} \times \mathbb{R}^{n}\right)$ we have

$$
\mathcal{F}_{p \rightarrow \xi_{p}} \mathcal{F}_{y \rightarrow p}^{-1}\left[K_{a}(x, y)\right]=K_{a}\left(x, \xi_{p}\right) .
$$

We also note from (12) that $\hat{S}$ can be trivially extended to $\mathcal{S}^{\prime}\left(\mathbb{R}^{2 n}\right)$. Hence, from (12)

$$
\begin{aligned}
\hat{S} \mathcal{F}_{y \rightarrow p}^{-1}\left[K_{a}(x, y)\right] & =\mathcal{F}_{\xi_{p} \rightarrow p}^{-1}\left[\hat{T} K_{a}\left(x, \xi_{p}\right)\right]=\mathcal{F}_{\xi_{p} \rightarrow p}^{-1}\left[K_{a}\left(x-\frac{\xi_{p}}{2}, x+\frac{\xi_{p}}{2}\right)\right] \\
& =\left(\frac{1}{2 \pi}\right)^{n / 2} \int_{\mathbb{R}^{n}} e^{-i p \cdot \xi_{p}} K_{a}\left(x+\frac{\xi_{p}}{2}, x-\frac{\xi_{p}}{2}\right) d \xi_{p} \\
& =\left(\frac{1}{2 \pi}\right)^{n / 2} a(x, p)
\end{aligned}
$$

which concludes the proof. 


\section{A Fractional generalization of the Wigner function and WEYL SYMBOLS}

In this section we use the metaplectic formulation of the Wigner function and Weyl symbols to obtain a natural fractional generalization for both these objects. We also extend the main properties of the Wigner transform and Weyl symbols to the fractional case. These results end up yielding simple, alternative proofs for several known results about the Weyl-Wigner formalism.

3.1. Main definitions and regularity results. A natural definition of the fractional cross Wigner function is

Definition 9. Let $\psi, \phi \in L^{2}\left(\mathbb{R}^{n}\right)$ and let $\hat{U}(\theta)$ be the unitary operator given by (12). The fractional cross Wigner function $W^{\theta}(\psi, \phi)$ is defined by

$$
W^{\theta}(\psi, \phi)(x, p):=(2 \pi)^{-n / 2} \hat{U}(\theta) \psi \otimes \overline{\hat{\phi}}(x, p)
$$

for $\theta \in \mathbb{R}$.

Since $\hat{U}(\theta)$ is periodic, the family of quasi-distributions $\left(W^{\theta}(\psi, \phi)\right)_{\theta \in \mathbb{R}}$ is also periodic. We have $W^{\theta}(\psi, \phi)=W^{\theta+\frac{2 \pi}{\sqrt{7}}}(\psi, \phi)$ for all $\theta \in \mathbb{R}$. Some important elements of this family are $W^{0}(\psi, \psi)=(2 \pi)^{-n / 2} \psi \otimes \overline{\hat{\psi}}$, which is $e^{i p \cdot x}$ times the anti-standard or Kirkwood quasi-distribution [20]. We also have $W^{2 \theta_{0}}(\psi, \psi)=(2 \pi)^{-n / 2} \bar{\psi} \otimes \hat{\psi}$ (check eq. (37)) which is $e^{-i p \cdot x}$ times the standard-ordered quasi-distribution [20]. Finally, $W^{\theta_{0}}(\psi, \phi)=W(\psi, \phi)$ is the cross Wigner function.

The metaplectic transformation $\hat{U}(\theta) \in \operatorname{Mp}(4 n)$ is a unitary operator $L^{2}\left(\mathbb{R}^{2 n}\right) \longrightarrow L^{2}\left(\mathbb{R}^{2 n}\right)$ and a continuous mapping $\mathcal{S}\left(\mathbb{R}^{2 n}\right) \longrightarrow \mathcal{S}\left(\mathbb{R}^{2 n}\right)$ that extends by duality to a continuous mapping $\mathcal{S}^{\prime}\left(\mathbb{R}^{2 n}\right) \longrightarrow \mathcal{S}^{\prime}\left(\mathbb{R}^{2 n}\right)$. Hence,

Theorem 10. The sesquilinear map $W^{\theta}$ is a continuous mapping of the forms:

$$
\begin{gathered}
W^{\theta}: \mathcal{S}\left(\mathbb{R}^{n}\right) \times \mathcal{S}\left(\mathbb{R}^{n}\right) \longrightarrow \mathcal{S}\left(\mathbb{R}^{2 n}\right) \\
W^{\theta}: L^{2}\left(\mathbb{R}^{n}\right) \times L^{2}\left(\mathbb{R}^{n}\right) \longrightarrow L^{2}\left(\mathbb{R}^{2 n}\right)
\end{gathered}
$$

and extends to a continuous mapping of the form

$$
W^{\theta}: \mathcal{S}^{\prime}\left(\mathbb{R}^{n}\right) \times \mathcal{S}^{\prime}\left(\mathbb{R}^{n}\right) \longrightarrow \mathcal{S}^{\prime}\left(\mathbb{R}^{2 n}\right)
$$

Proof. Let $M(\psi, \phi)=\psi \otimes \overline{\hat{\phi}}$. Since the Fourier transform $\mathcal{F}_{y \rightarrow p}$ is a continuous mapping $\mathcal{S}\left(\mathbb{R}^{n}\right) \longrightarrow \mathcal{S}\left(\mathbb{R}^{n}\right), L^{2}\left(\mathbb{R}^{n}\right) \longrightarrow L^{2}\left(\mathbb{R}^{n}\right)$ and extends by duality onto a continuous mapping $\mathcal{S}^{\prime}\left(\mathbb{R}^{n}\right) \longrightarrow \mathcal{S}^{\prime}\left(\mathbb{R}^{n}\right)$, the sesquilinear map $M$ can also be realized as a continuous mapping of any of the forms $\mathcal{S}\left(\mathbb{R}^{n}\right) \times \mathcal{S}\left(\mathbb{R}^{n}\right) \longrightarrow \mathcal{S}\left(\mathbb{R}^{2 n}\right), L^{2}\left(\mathbb{R}^{n}\right) \times L^{2}\left(\mathbb{R}^{n}\right) \longrightarrow L^{2}\left(\mathbb{R}^{2 n}\right)$ and also $\mathcal{S}^{\prime}\left(\mathbb{R}^{n}\right) \times$ $\mathcal{S}^{\prime}\left(\mathbb{R}^{n}\right) \longrightarrow \mathcal{S}^{\prime}\left(\mathbb{R}^{2 n}\right)$. The result follows from $W^{\theta}=(2 \pi)^{-n / 2} \hat{U}(\theta) M$. 
We also have for all $\theta \in \mathbb{R}$

$$
\left(W^{\theta}\left(\psi_{1}, \phi_{1}\right), W^{\theta}\left(\psi_{2}, \phi_{2}\right)\right)_{L^{2}\left(\mathbb{R}^{2 n}\right)}=\frac{1}{(2 \pi)^{n}}\left(\psi_{1}, \psi_{2}\right)_{L^{2}\left(\mathbb{R}^{n}\right)}\left(\phi_{1}, \phi_{2}\right)_{L^{2}\left(\mathbb{R}^{n}\right)}
$$

which is the fractional generalization of the Moyal identity. Hence, for $\phi_{1}=$ $\phi_{2}=\phi$ such that $\|\phi\|_{L^{2}\left(\mathbb{R}^{n}\right)}=1$, the fractional windowed Wigner transform $W_{\phi}^{\theta} \cdot=(2 \pi)^{n / 2} W^{\theta}(\cdot, \phi)$ is a non-surjective isometry $L^{2}\left(\mathbb{R}^{n}\right) \longrightarrow L^{2}\left(\mathbb{R}^{2 n}\right)$.

Just as in the case of the cross Wigner function, we may define a fractional generalization of Weyl symbols.

Definition 11. Let $\hat{a}: \mathcal{S}\left(\mathbb{R}^{n}\right) \longrightarrow \mathcal{S}^{\prime}\left(\mathbb{R}^{n}\right)$ be an arbitrary pseudo-differential operator and let $K_{a}(x, y) \in \mathcal{S}^{\prime}\left(\mathbb{R}^{n} \times \mathbb{R}^{n}\right)$ be its kernel. The $\theta$-Weyl symbol of $\hat{a}$ is defined by

$$
a^{\theta}(x, p):=(2 \pi)^{n / 2} \hat{U}(\theta) \mathcal{F}_{y \rightarrow p}^{-1}\left[K_{a}(x, y)\right] .
$$

As an example, let $a_{\hat{\rho}}^{\theta}$ be the $\theta$-Weyl symbol of the rank one operator $\hat{\rho}$ defined for fixed $\psi, \phi \in L^{2}\left(\mathbb{R}^{n}\right)$ by

$$
\hat{\rho}: \mathcal{S}\left(\mathbb{R}^{n}\right) \longrightarrow \mathcal{S}^{\prime}\left(\mathbb{R}^{n}\right), \xi \longmapsto \hat{\rho} \xi=(\xi, \phi)_{L^{2}\left(\mathbb{R}^{n}\right)} \psi .
$$

We have, as in the standard Weyl-Wigner formalism, $a_{\hat{\rho}}^{\theta}=(2 \pi)^{n} W^{\theta}(\psi, \phi)$.

3.2. Inversion and Kernel formulas. Let, as before,

$$
W_{\phi}^{\theta}: L^{2}\left(\mathbb{R}^{n}\right) \longrightarrow L^{2}\left(\mathbb{R}^{2 n}\right) ; \phi \longmapsto W_{\phi}^{\theta} \psi=(2 \pi)^{n / 2} W^{\theta}(\psi, \phi)
$$

be the $\theta$-windowed Wigner transform defined for $\theta \in \mathbb{R}$ and some window $\phi \in \mathcal{S}\left(\mathbb{R}^{n}\right)$ such that $\|\phi\|=1$. For $\theta=\theta_{0}$ we write $W_{\phi} \equiv W_{\phi}^{\theta_{0}}$ as in eq.(2). Let also $\left(W_{\phi}^{\theta}\right)^{*}: L^{2}\left(\mathbb{R}^{2 n}\right) \longrightarrow L^{2}\left(\mathbb{R}^{n}\right)$ be the mapping

$$
\left(W_{\phi}^{\theta}\right)^{*} \Psi(\cdot):=\int_{\mathbb{R}^{n}} \hat{\phi}(p) \hat{U}^{-1}(\theta) \Psi(\cdot, p) d p .
$$

Then

Theorem 12. The mapping $\left(W_{\phi}^{\theta}\right)^{*}$ is the adjoint of $W_{\phi}^{\theta}$ and satisfies:

(i) $\left(W_{\phi}^{\theta}\right)^{*} W_{\phi}^{\theta}=1$;

(ii) $W_{\phi}^{\theta}\left(W_{\phi}^{\theta}\right)^{*}=P_{\phi}^{\theta}$ where $P_{\phi}^{\theta}$ is the orthogonal projection onto the range of $W_{\phi}^{\theta}$; by

(iii) $\left(W_{\phi}^{\theta}\right)^{*}$ extends to a continuous operator $\mathcal{S}^{\prime}\left(\mathbb{R}^{2 n}\right) \longrightarrow \mathcal{S}^{\prime}\left(\mathbb{R}^{n}\right)$ defined

$$
\left\langle\left(W_{\phi}^{\theta}\right)^{*} \Psi, \xi\right\rangle=\left\langle\Psi, \hat{U}^{-1}(\theta) \xi \otimes \hat{\phi}\right\rangle, \quad \forall \xi \in \mathcal{S}\left(\mathbb{R}^{n}\right) .
$$

Proof. The adjoint of $W_{\phi}^{\theta}$ is defined by

$$
\left(\left(W_{\phi}^{\theta}\right)^{*} \Psi, \xi\right)=\left(\Psi, W_{\phi}^{\theta} \xi\right), \quad \forall \xi \in L^{2}\left(\mathbb{R}^{n}\right)
$$


and since

$$
\begin{aligned}
\left(\Psi, W_{\phi}^{\theta} \xi\right) & =(\Psi, \hat{U}(\theta) \xi \otimes \overline{\hat{\phi}})=\left(\hat{U}^{-1}(\theta) \Psi, \xi \otimes \overline{\hat{\phi}}\right) \\
& =\int_{\mathbb{R}^{n}} \bar{\xi}(x)\left[\int_{\mathbb{R}^{n}} \hat{\phi}(p) \hat{U}^{-1}(\theta) \Psi(x, p) d p\right] d x
\end{aligned}
$$

we have

$$
\left(W_{\phi}^{\theta}\right)^{*} \Psi=\int_{\mathbb{R}^{n}} \hat{\phi}(p) \hat{U}^{-1}(\theta) \Psi(x, p) d p
$$

as claimed.

(i) Let now $\Psi=W_{\phi}^{\theta} \psi=\hat{U}(\theta)(\psi \otimes \overline{\hat{\phi}})$. Then

$$
\begin{aligned}
\left(W_{\phi}^{\theta}\right)^{*} \Psi & =\int_{\mathbb{R}^{n}} \hat{\phi}(p) \hat{U}^{-1}(\theta) \hat{U}(\theta)(\psi \otimes \overline{\hat{\phi}})(x, p) d p \\
& =\int_{\mathbb{R}^{n}} \hat{\phi}(p) \overline{\hat{\phi}}(p) \psi(x) d p=\psi(x) .
\end{aligned}
$$

(ii) Conversely, let $\Psi \in L^{2}\left(\mathbb{R}^{2 n}\right)$ and let $P_{\phi}^{\theta}=W_{\phi}^{\theta}\left(W_{\phi}^{\theta}\right)^{*}$. Then, of course

$$
P_{\phi}^{\theta} P_{\phi}^{\theta}=W_{\phi}^{\theta}\left(W_{\phi}^{\theta}\right)^{*} W_{\phi}^{\theta}\left(W_{\phi}^{\theta}\right)^{*}=P_{\phi}^{\theta}
$$

and if $\Psi \in \operatorname{Ran} W_{\phi}^{\theta}$ then $\Psi=W_{\phi}^{\theta} \psi$ for some $\psi \in L^{2}\left(\mathbb{R}^{n}\right)$ and

$$
P_{\phi}^{\theta} \Psi=W_{\phi}^{\theta}\left(W_{\phi}^{\theta}\right)^{*} W_{\phi}^{\theta} \psi=W_{\phi}^{\theta} \psi=\Psi .
$$

Hence, the claim.

(iii) As a distribution $\left(W_{\phi}^{\theta}\right)^{*} \Psi$ is completely defined by its action on arbitrary test functions $\xi \in \mathcal{S}\left(\mathbb{R}^{n}\right)$. For $\Psi \in \mathcal{S}\left(\mathbb{R}^{2 n}\right)$ and $\phi \in \mathcal{S}\left(\mathbb{R}^{n}\right)$, we have from (25)

$$
\left\langle\left(W_{\phi}^{\theta}\right)^{*} \Psi, \xi\right\rangle=\left\langle\hat{U}^{-1}(\theta) \Psi, \xi \otimes \hat{\phi}\right\rangle=\left\langle\Psi, \hat{U}^{-1}(\theta) \xi \otimes \hat{\phi}\right\rangle .
$$

Since $\hat{U}^{-1}(\theta): \mathcal{S}^{\prime}\left(\mathbb{R}^{2 n}\right) \longrightarrow \mathcal{S}^{\prime}\left(\mathbb{R}^{2 n}\right)$, this formula extends trivially to $\Psi \in$ $\mathcal{S}^{\prime}\left(\mathbb{R}^{2 n}\right)$.

All these considerations are of course valid for the standard windowed Wigner transform, which is just a particular element of the family $W_{\phi}^{\theta}$, $\theta \in \mathbb{R}$.

To proceed let us generalize the Kernel formula (20) for the fractional case. Let the kernel of $\hat{a}: \mathcal{S}\left(\mathbb{R}^{n}\right) \longrightarrow \mathcal{S}^{\prime}\left(\mathbb{R}^{n}\right)$ be given by $K_{a}(x, y) \in \mathcal{S}^{\prime}\left(\mathbb{R}^{n} \times \mathbb{R}^{n}\right)$ so that

$$
\hat{a} \psi(x)=\left\langle K_{a}(x, y), \psi(y)\right\rangle
$$

We then have

$$
\langle\hat{a} \psi, \bar{\phi}\rangle=\left\langle K_{a}(x, y), \psi(y) \bar{\phi}(x)\right\rangle
$$

and it is well known that this formula can be re-expressed in terms of the Wigner function as in (20). This is because

$$
\left\langle K_{a}(x, y), \psi(y) \bar{\phi}(x)\right\rangle=\langle a(x, p), W(\psi, \phi)(x, p)\rangle .
$$


Let also $\hat{b}=\hat{a}^{\dagger}$ be the formal adjoint of $\hat{a}$, defined by

$$
\langle\hat{b} \psi, \bar{\phi}\rangle=\overline{\langle\hat{a} \phi, \bar{\psi}\rangle} \quad, \quad \forall \psi, \phi \in \mathcal{S}\left(\mathbb{R}^{n}\right) .
$$

It is well known that $K_{b}(x, y)=\overline{K_{a}}(y, x)$. This relation follows immediately from eq.(29) by taking into account that

$$
\langle\hat{b} \psi, \bar{\phi}\rangle=\left\langle K_{b}(x, y), \psi(y) \bar{\phi}(x)\right\rangle
$$

and that

$$
\overline{\langle\hat{a} \phi, \bar{\psi}\rangle}=\left\langle\overline{K_{a}}(y, x), \psi(y) \bar{\phi}(x)\right\rangle .
$$

We can now state the following

Theorem 13. Let $\hat{a}: \mathcal{S}\left(\mathbb{R}^{n}\right) \longrightarrow \mathcal{S}^{\prime}\left(\mathbb{R}^{n}\right)$ be an arbitrary pseudo-differential operator and let $\hat{b}$ be its formal adjoint. Let $b^{\theta}$ be the $\theta$-Weyl symbol of $\hat{b}$. Then

$$
\langle\hat{a} \psi, \bar{\phi}\rangle=\left\langle\overline{b^{\theta}}, W^{\theta}(\psi, \phi)\right\rangle
$$

for all $\psi, \phi \in \mathcal{S}\left(\mathbb{R}^{n}\right)$.

Proof. We have from (27)

$$
\begin{aligned}
\langle\hat{a} \psi, \bar{\phi}\rangle & =\overline{\left\langle\overline{K_{a}}(y, x), \overline{\psi(x) \bar{\phi}(y)}\right\rangle} \\
& =\overline{\left\langle\hat{U}(\theta) \mathcal{F}_{y \rightarrow p}^{-1}\left[\overline{K_{a}}(y, x)\right], \overline{\hat{U}(\theta) \mathcal{F}_{y \rightarrow p}^{-1}[\psi(x) \bar{\phi}(y)]}\right.} \\
& =(2 \pi)^{n / 2}\left\langle\overline{\hat{U}(\theta) \mathcal{F}_{y \rightarrow p}^{-1}\left[\overline{K_{a}}(y, x)\right]}, W^{\theta}(\psi, \phi)(x, p)\right\rangle .
\end{aligned}
$$

To complete the proof we just notice that $K_{b}(x, y)=\overline{K_{a}}(y, x)$ and use the definition of $\theta$-Weyl symbol (23).

Finally, notice that in the Weyl case $\theta=\theta_{0}$ we have $\overline{b^{\theta_{0}}}=a^{\theta_{0}}=a$ and so formula (30) reduces to the standard formula (20).

3.3. $\theta$-Weyl calculus and quantization. From the definitions 9 and 11 we immediately realize that

$$
\begin{aligned}
W^{\theta}(\psi, \phi) & =\hat{U}\left(\theta-\theta_{0}\right) W(\psi, \phi) \\
a^{\theta} & =\hat{U}\left(\theta-\theta_{0}\right) a .
\end{aligned}
$$

where $a \equiv a^{\theta_{0}}$ and $a^{\theta}$ are the Weyl and the $\theta$-Weyl symbols of an arbitrary pseudo-differential operator $\hat{a}$, respectively. These formulas allow us to relate the standard and the fractional Weyl calculus. Let us denote by $\hat{a} \stackrel{\theta}{\longleftrightarrow} a^{\theta}$ the association between the $\theta$-symbol $a^{\theta}=\hat{U}\left(\theta-\theta_{0}\right) a$ and the pseudo-differential operator $\hat{a} \stackrel{\text { Weyl }}{\longleftrightarrow} a$. Then

Theorem 14. Let $\hat{a}: \mathcal{S}\left(\mathbb{R}^{n}\right) \longrightarrow \mathcal{S}^{\prime}\left(\mathbb{R}^{n}\right)$ and $\hat{b}: \mathcal{S}\left(\mathbb{R}^{n}\right) \longrightarrow \mathcal{S}\left(\mathbb{R}^{n}\right)$ be two arbitrary pseudo-differential operators. Let $\hat{a} \stackrel{\theta}{\longleftrightarrow} a^{\theta}$ and $\hat{b} \stackrel{\theta}{\longleftrightarrow} b^{\theta}$. Then

$$
\hat{a} \hat{b} \stackrel{\theta}{\longleftrightarrow} a^{\theta} *_{\theta} b^{\theta}
$$


where the product $*_{\theta}$ is given by

$$
a^{\theta} *_{\theta} b^{\theta}=\hat{U}\left(\theta-\theta_{0}\right)\left[\left(\hat{U}^{-1}\left(\theta-\theta_{0}\right) a^{\theta}\right) *_{M}\left(\hat{U}^{-1}\left(\theta-\theta_{0}\right) b^{\theta}\right)\right] .
$$

Here $*_{M}$ is the standard Moyal product [16]

$$
a *_{M} b(z)=\left(\frac{1}{4 \pi}\right)^{2 n} \int_{\mathbb{R}^{4 n}} e^{\frac{i}{2} \sigma\left(z^{\prime}, z^{\prime \prime}\right)} a\left(z+\frac{1}{2} z^{\prime}\right) b\left(z-\frac{1}{2} z^{\prime \prime}\right) d z^{\prime} d z^{\prime \prime}
$$

where $z=(x, p)$ are canonical coordinates, and $\sigma$ is the standard symplectic form on $\mathbb{R}^{2 n}$.

Proof. Let $\hat{a} \stackrel{\text { Weyl }}{\longleftrightarrow} a$ and $\hat{b} \stackrel{\text { Weyl }}{\longleftrightarrow} b$. Then [16]

$$
\hat{a} \hat{b} \stackrel{\text { Weyl }}{\longleftrightarrow} a *_{M} b .
$$

The association between an arbitrary $\hat{a}$ and its $\theta$-symbol $a^{\theta}$ is defined by $\hat{a} \stackrel{\theta}{\longleftrightarrow} a^{\theta}=\hat{U}\left(\theta-\theta_{0}\right) a$ where $\hat{a} \stackrel{\text { Weyl }}{\longleftrightarrow} a$. Hence, the $\theta$-symbol of $\hat{a} \hat{b}$ is

$$
a^{\theta} *_{\theta} b^{\theta}=\hat{U}\left(\theta-\theta_{0}\right)\left(a *_{M} b\right) .
$$

Using $a=\hat{U}^{-1}\left(\theta-\theta_{0}\right) a^{\theta}$ and $b=\hat{U}^{-1}\left(\theta-\theta_{0}\right) b^{\theta}$ we get (32).

It easily follows from (32) that $*_{\theta}$ is non-commutative, distributive and associative. Let us prove this last property. Since $*_{M}$ is associative we have (let $\left.\hat{U}=\hat{U}\left(\theta-\theta_{0}\right)\right)$

$$
\begin{aligned}
\left(a^{\theta} *_{\theta} b^{\theta}\right) *_{\theta} c^{\theta} & =\hat{U}\left[\left[\left(\hat{U}^{-1} a^{\theta}\right) *_{M}\left(\hat{U}^{-1} b^{\theta}\right)\right] *_{M}\left(\hat{U}^{-1} c^{\theta}\right)\right] \\
& =\hat{U}\left[\left(\hat{U}^{-1} a^{\theta}\right) *_{M}\left[\left(\hat{U}^{-1} b^{\theta}\right) *_{M}\left(\hat{U}^{-1} c^{\theta}\right)\right]\right] \\
& =a^{\theta} *_{\theta}\left(b^{\theta} *_{\theta} c^{\theta}\right) .
\end{aligned}
$$

To proceed let us determine the $\theta$-symbol of the adjoint operator. Let, as before, $\hat{b}=\hat{a}^{\dagger}$ be the formal adjoint of $\hat{a}$. Then the Weyl symbols of $\hat{a}$ and $\hat{b}$ satisfy $a=\bar{b}$ and we have for the $\theta$-symbols

$$
b^{\theta_{0}+\alpha}=\hat{U}(\alpha) b=\hat{U}(\alpha) \bar{a}=\overline{\hat{U}^{-1}(\alpha) a}=\overline{a^{\theta_{0}-\alpha}} .
$$

Hence, and in general, the $\theta$-symbol of a self-adjoint operator is not real. The exception is the standard Weyl case.

Using the result (34) we can re-write the kernel formula (30) in the form

$$
\langle\hat{a} \psi, \bar{\phi}\rangle=\left\langle a^{\theta_{0}-\alpha}, W^{\theta_{0}+\alpha}(\psi, \phi)\right\rangle
$$

for all $\alpha \in \mathbb{R}$.

Finally, let us remark on a few features of the fractional Weyl quantization. The next result is a trivial consequence of (35)

Corollary 15. The expectation value of a quantum observable $\hat{a}$ in a state $\psi \in \mathcal{S}\left(\mathbb{R}^{n}\right)$ is given in terms of its $\theta$-symbol by

$$
\langle\hat{a} \psi, \bar{\psi}\rangle=\int_{\mathbb{R}^{2 n}} \overline{a^{\theta}(z)} W^{\theta}(\psi, \psi)(z) d z .
$$


where the integral is interpreted in the distributional sense.

Proof. The proof follows from eqs.(34) and (35) by noticing that for $\hat{a}$ selfadjoint we have $\hat{b}=\hat{a}$ and so $a^{\theta_{0}-\alpha}=\overline{a^{\theta_{0}+\alpha}}$. Notice also that in the standard Weyl case, we have in addition $a^{\theta_{0}} \equiv a=\bar{a}$.

From eq.(35) we can also determine the marginal probability distributions for the fractional Wigner function. Let, for instance, $\hat{a}$ be the projection operator given formally by $\left|x_{0}><x_{0}\right|$ where $x_{0} \in \mathbb{R}^{n}$. We have explicitly

$$
\hat{a}: \mathcal{S}\left(\mathbb{R}^{n}\right) \longrightarrow \mathcal{S}^{\prime}\left(\mathbb{R}^{n}\right) ; \psi \longmapsto \psi\left(x_{0}\right) \delta\left(x-x_{0}\right)
$$

where $\delta$ is the Dirac delta distribution. The Weyl symbol of $\hat{a}$ can be easily calculated from eq.(18): $a(x, p)=\delta\left(x-x_{0}\right)$. Hence, the marginal probability distribution for the position observable is simply

$$
\begin{aligned}
\mathcal{P}\left(x_{0}\right)=\langle\hat{a} \psi, \bar{\psi}\rangle & =\int_{\mathbb{R}^{n}} \int_{\mathbb{R}^{n}} \delta\left(x-x_{0}\right) W(\psi, \psi)(x, p) d x d p \\
& =\int_{\mathbb{R}^{n}} W(\psi, \psi)\left(x_{0}, p\right) d p .
\end{aligned}
$$

In the fractional case, we also have from eq.(31) and eq.(36)

$$
\mathcal{P}\left(x_{0}\right)=\int_{\mathbb{R}^{n}} \int_{\mathbb{R}^{n}}\left(\hat{U}^{-1}\left(\theta-\theta_{0}\right) \delta\left(x-x_{0}\right)\right) W^{\theta}(\psi, \psi)(x, p) d x d p .
$$

However, in general, this formula does not further simplify as in the case of standard Wigner functions.

Another important feature of the Wigner function $W(\psi, \psi)$ is that it is a real quasidistribution. This property is also not shared by the fractional Wigner functions. For the $\theta$-cross Wigner function we get from (31)

$$
W^{\theta_{0}+\alpha}(\psi, \phi)=\overline{W^{\theta_{0}-\alpha}(\phi, \psi)}
$$

which yields a reality condition only for the case $\alpha=0$ and $\phi=\psi$.

\section{The Bopp RePresentation of QUANTUm MeCHANiCS}

In this section we show that the windowed Wigner transform $W_{\phi}$ intertwines the Schrödinger and the Bopp representations of quantum mechanics and discuss the implications of this result for the spectral and dynamical properties of operators in the two representations. This subject was previously studied in several papers $9,17,18$. Here, we present an alternative approach using the metaplectic formalism developed in the previous sections.

Let $\hat{a}: \mathcal{S}\left(\mathbb{R}^{n}\right) \longrightarrow \mathcal{S}^{\prime}\left(\mathbb{R}^{n}\right)$ be a generic pseudo-differential operator. In terms of its Weyl symbol $a \in \mathcal{S}^{\prime}\left(\mathbb{R}^{n} \times \mathbb{R}^{n}\right)$, the operator can be written [24]

$$
\hat{a} \psi(x)=\frac{1}{(2 \pi)^{n}} \int_{\mathbb{R}^{2 n}} e^{i\left(x-x^{\prime}\right) \cdot \xi_{x}} a\left(\frac{1}{2}\left(x+x^{\prime}\right), \xi_{x}\right) \psi\left(x^{\prime}\right) d x^{\prime} d \xi_{x}
$$

where the integral is well defined for $a \in \mathcal{S}\left(\mathbb{R}^{n} \times \mathbb{R}^{n}\right)$ and should otherwise be interpreted in the distributional sense. The operator $\hat{a}$ is formally $\hat{a}=a\left(x,-i \partial_{x}\right)$ and the mapping $a \longmapsto \hat{a}$ yields a precise definition of the 
standard Schrödinger representation of quantum mechanics.

A trivial extension of $\hat{a}$ to phase space functions is given by the operator $\hat{A}: \mathcal{S}\left(\mathbb{R}^{n} \times \mathbb{R}^{n}\right) \longrightarrow \mathcal{S}^{\prime}\left(\mathbb{R}^{n} \times \mathbb{R}^{n}\right)$ of the form:

$$
\hat{A} \Psi(x, p)=\frac{1}{(2 \pi)^{n}} \int_{\mathbb{R}^{2 n}} e^{i\left(x-x^{\prime}\right) \cdot \xi_{x}} a\left(\frac{1}{2}\left(x+x^{\prime}\right), \xi_{x}\right) \Psi\left(x^{\prime}, p\right) d x^{\prime} d \xi_{x}
$$

Then

Theorem 16. The operator $\hat{A}$ is a pseudo-differential operator with Weyl symbol

$$
A\left(x, p ; \xi_{x}, \xi_{p}\right)=a\left(x, \xi_{x}\right) .
$$

Moreover, for an arbitrary wave function $\Psi(x, p)=\psi \otimes \overline{\hat{\phi}}(x, p) \in \mathcal{S}\left(\mathbb{R}^{2 n}\right)$, we have

$$
\hat{A}(\psi \otimes \overline{\hat{\phi}})=(\hat{a} \psi) \otimes \overline{\hat{\phi}}
$$

Proof. Let $\hat{A}$ be the pseudo-differential operator with Weyl symbol $A$ of the form (40). Then $\hat{A} \Psi(x, p)$ is explicitly

$$
\begin{aligned}
& \hat{A} \Psi(x, p) \\
= & \frac{1}{(2 \pi)^{2 n}} \int_{\mathbb{R}^{4 n}} e^{i\left[\left(x-x^{\prime}\right) \cdot \xi_{x}+\left(p-p^{\prime}\right) \cdot \xi_{p}\right]} A\left(\frac{x+x^{\prime}}{2}, \frac{p+p^{\prime}}{2}, \xi_{x}, \xi_{p}\right) \Psi\left(x^{\prime}, p^{\prime}\right) d x^{\prime} d p^{\prime} d \xi_{x} d \xi_{p} \\
= & \frac{1}{(2 \pi)^{2 n}} \int_{\mathbb{R}^{4 n}} e^{i\left[\left(x-x^{\prime}\right) \cdot \xi_{x}+\left(p-p^{\prime}\right) \cdot \xi_{p}\right]} a\left(\frac{1}{2}\left(x+x^{\prime}\right), \xi_{x}\right) \Psi\left(x^{\prime}, p^{\prime}\right) d x^{\prime} d p^{\prime} d \xi_{x} d \xi_{p} \\
= & \frac{1}{(2 \pi)^{n}} \int_{\mathbb{R}^{2 n}} e^{i\left(x-x^{\prime}\right) \cdot \xi_{x}} a\left(\frac{1}{2}\left(x+x^{\prime}\right), \xi_{x}\right) \Psi\left(x^{\prime}, p\right) d x^{\prime} d \xi_{x}
\end{aligned}
$$

which coincides with the expression (39) exactly.

The intertwining relation (41) follows directly from (39) for $\Psi(x, p)=$ $\psi(x) \overline{\hat{\phi}}(p)$.

Just like $\hat{a}$, the operator $\hat{A}$ is also formally $\hat{A}=a\left(x,-i \partial_{x}\right)$ (but now the fundamental operators $x$. and $-i \partial_{x}$ act on phase space functions). The mapping $a \longmapsto \hat{A}$ yields the phase space Schrödinger representation of quantum mechanics. The spectral and dynamical properties of the operators $\hat{a}$ and $\hat{A}$ are equivalent. This is an important property that we now discuss in some detail. For complete proofs the reader should refer to [9].

For an arbitrary window $\phi \in \mathcal{S}\left(\mathbb{R}^{n}\right)$ such that $\|\phi\|_{L^{2}\left(\mathbb{R}^{n}\right)}=1$, let

$$
T_{\phi}: \mathcal{S}^{\prime}\left(\mathbb{R}^{n}\right) \longrightarrow \mathcal{S}^{\prime}\left(\mathbb{R}^{2 n}\right) ; \psi \longmapsto \Psi=T_{\phi} \psi=\psi \otimes \overline{\hat{\phi}},
$$

and let

$$
T_{\phi}^{*}: \mathcal{S}^{\prime}\left(\mathbb{R}^{2 n}\right) \longrightarrow \mathcal{S}^{\prime}\left(\mathbb{R}^{n}\right) ; \Psi \longmapsto \psi=T_{\phi}^{*} \Psi
$$

be defined by

$$
\left\langle T_{\phi}^{*} \Psi, \xi\right\rangle=\langle\Psi, \xi \otimes \hat{\phi}\rangle \quad, \quad \forall \xi \in \mathcal{S}\left(\mathbb{R}^{n}\right)
$$


These two maps coincide exactly with $W_{\phi}^{\theta}$ and $\left(W_{\phi}^{\theta}\right)^{*}$ (given by eq.(24) and eq.(26), respectively) for $\theta=0$. Hence, they satisfy the properties stated in Theorem 12 (i) and (ii). They also satisfy the intertwining relations

$$
T_{\phi} \hat{a}=\hat{A} T_{\phi} \quad, \quad T_{\phi}^{*} \hat{A}=\hat{a} T_{\phi}^{*} .
$$

The first relation is valid in $\mathcal{S}\left(\mathbb{R}^{n}\right)$ and is just a restatement of eq.(41). The second relation is valid in $\mathcal{S}\left(\mathbb{R}^{2 n}\right)$ and was proved in $[9$.

The next theorem is a consequence of these intertwining relations. It shows that the operators $\hat{a}$ and $\hat{A}$ have equivalent spectral properties. The proof can also be found in [9].

Theorem 17. Let $\hat{a}$ and $\hat{A}$ be the pseudo-differential operators given by eqs. (38,39), respectively. Let $\phi \in \mathcal{S}\left(\mathbb{R}^{n}\right)$ be such that $\|\phi\|_{L^{2}\left(\mathbb{R}^{n}\right)}=1$. Then

(i) The eigenvalues of $\hat{a}$ and $\hat{A}$ are the same.

(ii) If $\psi_{\lambda}$ is an eigenfunction of $\hat{a}$ then $\Psi_{\lambda}=T_{\phi} \psi_{\lambda}$ is an eigenfunction of $\hat{A}$ (associated with the same eigenvalue).

(iii) Conversely, let $\Psi_{\lambda}$ be an eigenfunction of $\hat{A}$. If $\psi_{\lambda}=T_{\phi}^{*} \Psi_{\lambda} \neq 0$ then $\psi_{\lambda}$ is an eigenfunction of $\hat{a}$ (associated with the same eigenvalue).

(iv) If $\left(\psi_{\lambda}\right)_{\lambda}$ is an orthonormal basis of eigenfunctions of $\hat{a}$ and $\left(\phi_{\gamma} \in\right.$ $\left.\mathcal{S}\left(\mathbb{R}^{n}\right)\right)_{\gamma}$ is an orthonormal basis of $L^{2}\left(\mathbb{R}^{n}\right)$ then $\left(T_{\phi_{\gamma}} \psi_{\lambda}\right)_{\gamma, \lambda}$ is a complete set of eigenfunctions of $\hat{A}$ and forms an orthonormal basis of $L^{2}\left(\mathbb{R}^{2 n}\right)$.

The next theorem concerns the dynamical properties.

Theorem 18. Let $\hat{a}$ and $\hat{A}$ be given by eqs. (38), respectively. Let $\phi \in$ $\mathcal{S}\left(\mathbb{R}^{n}\right)$ satisfy $\|\phi\|_{L^{2}\left(\mathbb{R}^{n}\right)}=1$. Then:

(i) If $\psi(x, t)$ is the solution of the initial value problem

$$
i \frac{\partial \psi}{\partial t}=\hat{a} \psi \quad, \quad \psi(\cdot, 0)=\psi_{0}(\cdot) \in \mathcal{S}\left(\mathbb{R}^{n}\right)
$$

then $\Psi=T_{\phi} \psi$ is the solution of

$$
i \frac{\partial \Psi}{\partial t}=\hat{A} \Psi \quad, \quad \Psi(\cdot, 0)=\Psi_{0}(\cdot) \in \mathcal{S}\left(\mathbb{R}^{2 n}\right)
$$

where $\Psi_{0}=T_{\phi} \psi_{0}$.

(ii) Conversely, if $\Psi$ is the solution of the initial value problem (44) then $\psi=T_{\phi}^{*} \Psi$ is the solution of 43 . .

The proof follows directly from the intertwining relations and the fact that the time derivative operator commutes with both $T_{\phi}$ and $T_{\phi}^{*}[9]$.

The results of the previous theorems can be extended to any other operator that is unitarily related with $\hat{A}$. This is the case of the Bopp operators, formally defined by the mapping

$$
a\left(x, \xi_{x}\right) \longmapsto \hat{A}_{B}=a\left(x+\frac{i}{2} \partial_{p}, p-\frac{i}{2} \partial_{x}\right) .
$$


This map attributes to each phase space symbol $a \in \mathcal{S}^{\prime}\left(\mathbb{R}^{2 n}\right)$, an operator $\hat{A}_{B}$ acting on wave functions $\Psi(x, p)$ with support on the phase space. The Bopp representation is closely related with the deformation quantization of Bayen et. al [1, 2, 10, 11] and was used, in this context, to prove some general spectral results for the stargenvalue equation [9, 17].

We now prove that the Bopp representation can be precisely defined by the mapping

$$
a \longmapsto \hat{A}_{B}=\hat{S} \hat{A} \hat{S}^{-1}
$$

where $a \in \mathcal{S}^{\prime}\left(\mathbb{R}^{2 n}\right), \hat{A}$ is given by eq. (39) and $\hat{S}=\hat{U}\left(\theta_{0}\right)$ by eq.(12). Let us consider first the general result

Proposition 19 (Metaplectic covariance property). Let $\hat{A} \stackrel{\text { Weyl }}{\longleftrightarrow} A$ be a pseudo-differential operator with Weyl symbol $A \in \mathcal{S}^{\prime}\left(\mathbb{R}^{4 n}\right)$, let $\hat{S} \in M p(4 n)$ and let $S \in S p(4 n)$ be its natural projection onto $S p(4 n)$. Then

$$
\hat{A}_{S}=\hat{S} \hat{A} \hat{S}^{-1} \stackrel{\text { Weyl }}{\longleftrightarrow} A_{S}=A \circ S^{-1} .
$$

For a proof see [16, 25]. We then have:

Theorem 20. The operators $\hat{A}_{B}$ (given by eq. 46 ) are pseudo-differential operators with Weyl symbols

$$
A_{B}\left(x, p ; \xi_{x}, \xi_{p}\right)=a\left(x-\frac{\xi_{p}}{2}, p+\frac{\xi_{x}}{2}\right) .
$$

Proof. It follows from Proposition 19 and the definition of $\hat{A}_{B}$ (46) that $\hat{A}_{B} \stackrel{\text { Weyl }}{\longleftrightarrow} A_{B}=A \circ S^{-1}$ where $A \stackrel{\text { Weyl }}{\longleftrightarrow} \hat{A}$ is given by (40) and $S^{-1}$ is the inverse of the projection of $\hat{S}$ onto $\operatorname{Sp}(4 n)$. Hence $S^{-1}=s\left(-\theta_{0}\right)$ (cf. Theorem 4) which can be easily determined from (8) and (9). We have

$$
S^{-1}: \mathbb{R}^{4 n} \longrightarrow \mathbb{R}^{4 n}, \quad\left\{\begin{array}{l}
x \longmapsto x-\xi_{p} / 2 \\
\xi_{x} \longmapsto \xi_{x} / 2+p \\
p \longmapsto p-\xi_{x} / 2 \\
\xi_{p} \longmapsto \xi_{p} / 2+x
\end{array}\right.
$$

The result (48) then follows from $A_{B}=A \circ S^{-1}$ by taking into account (40).

For the fundamental operators the mapping (46) yields:

$$
\begin{gathered}
a\left(x, \xi_{x}\right)=x \Longrightarrow \hat{A}=x \cdot \Longrightarrow \hat{A}_{B}=x+\frac{i}{2} \partial_{p} \\
a\left(x, \xi_{x}\right)=\xi_{x} \Longrightarrow \hat{A}=-i \partial_{x} \Longrightarrow \hat{A}_{B}=p-\frac{i}{2} \partial_{x}
\end{gathered}
$$

and more generally, we have formally

$$
a\left(x, \xi_{x}\right) \longmapsto \hat{A}_{B}=a\left(x+\frac{i}{2} \partial_{p}, p-\frac{i}{2} \partial_{x}\right) .
$$


Finally, it follows from $\hat{A}_{B}=\hat{S} \hat{A} \hat{S}^{-1}$ that the spectral and dynamical properties of the Bopp and the phase space Schrödinger representations are equivalent. In view of Theorems 17, 18 this equivalence can be extended to the standard Schrödinger representation. Combining (42) with $\hat{A}_{B}=\hat{S} \hat{A} \hat{S}^{-1}$ we obtain the intertwining relations

$$
\hat{A}_{B} \hat{S} T_{\phi}=\hat{S} T_{\phi} \hat{a} \quad, \quad T_{\phi}^{*} \hat{S}^{-1} \hat{A}_{B}=\hat{a} T_{\phi}^{*} \hat{S}^{-1}
$$

which can be re-written in terms of the windowed Wigner transform $W_{\phi} \equiv$ $W_{\phi}^{\theta_{0}}$ and its adjoint $W_{\phi}^{*} \equiv\left(W_{\phi}^{\theta_{0}}\right)^{*}$ (given respectively by (24) and (25]26) for $\theta=\theta_{0}$ )

$$
\hat{A}_{B} W_{\phi}=W_{\phi} \hat{a} \quad, \quad W_{\phi}^{*} \hat{A}_{B}=\hat{a} W_{\phi}^{*} .
$$

The Theorems 17 and 18 are then valid ipsis verbis for the pair of operators $\hat{a}$ and $\hat{A}_{B}$ if we substitute the maps $T_{\phi}$ and $T_{\phi}^{*}$ by the maps $W_{\phi}$ and $W_{\phi}^{*}$, respectively.

Acknowledgements. We thank the anonymous referee for a detailed reading of the paper and for many useful insights and remarks. Maurice de Gosson has been financed by the Austrian Research Agency FWF (Projektnummer P20442-N13). Nuno Costa Dias and João Nuno Prata have been supported by the grant PTDC/MAT/099880/2008 of the Portuguese Science Foundation (FCT).

\section{REFERENCES}

[1] F. Bayen, M. Flato, C. Fronsdal, A. Lichnerowicz, and D. Sternheimer, Deformation Theory and Quantization. I. Deformation of Symplectic Structures, Annals of Physics, 110 (1978), 111-151.

[2] F. Bayen, M. Flato, C. Fronsdal, A. Lichnerowicz, and D. Sternheimer, Deformation Theory and Quantization. II. Physical Applications, Annals of Physics, 111 (1978), $6-110$.

[3] D.I. Bondar, R. Cabrera, R.R. Lompay, M.Y. Ivanov and H.A. Rabitz, Operational dynamical modeling transcending quantum and classical mechanics, Phys. Rev. Lett. 109, 190403 (2012)

[4] F. Bopp. La mécanique quantique est-elle une mécanique statistique particulière? Ann. Inst. H. Poincaré, 15 (1956), 81-112

[5] A. Bracken and P. Watson, The quantum state vector in phase space and Gabor's windowed Fourier transform, J. Phys. A: Math. Theor. 43 (2010) 395304 (19pp)

[6] N.C. Dias, Half-Quantization, J. Phys. A: Math. Gen. 34 (2001) 771-791.

[7] N.C. Dias, M. de Gosson, F. Luef, J.N. Prata, A Deformation Quantization Theory for Non-Commutative Quantum Mechanics, J. Math. Phys. 51 (2010) 072101 (12 pages).

[8] N.C. Dias, M. de Gosson, F. Luef, J.N. Prata, A pseudo-differential calculus on nonstandard symplectic space; spectral and regularity results in modulation spaces. J. Math. Pure Appl. 96 (2011) 423-445

[9] N.C. Dias, M. de Gosson, F. Luef, J.N. Prata, Quantum mechanics in phase space: the Schrödinger and the Moyal representations. Journal of Pseudo-Differential Operators and Applications, 3(4) (2012) 367-398. 
[10] N.C. Dias, J.N. Prata, Formal solutions of stargenvalue equations, Ann. Phys.(N. Y.), 311 (2004) 120-151.

[11] N.C. Dias, J.N. Prata, Admissible states in quantum phase space, Ann. Phys.(N. Y.), 313 (2004) 110-146.

[12] G. B. Folland. Harmonic Analysis in Phase space, Annals of Mathematics studies, Princeton University Press, Princeton, N.J. (1989)

[13] M. de Gosson. Maslov Classes, Metaplectic Representation and Lagrangian Quantization, Research Notes in Mathematics, 95, Wiley-VCH, Berlin, (1997)

[14] M. de Gosson. Symplectic Geometry and Quantum Mechanics, Birkhäuser, Basel, series "Operator Theory: Advances and Applications" (subseries: "Advances in Partial Differential Equations"), Vol. 166 (2006)

[15] M. de Gosson. Spectral Properties of a Class of Generalized Landau Operators, Comm. Partial Differential Operators, 33(11) (2008), 2096-2104

[16] M. de Gosson. Symplectic Methods in Harmonic Analysis and in Mathematical Physics; Birkhäuser, series "Pseudo-Differential Operators, Theory and Applications" 2011

[17] M. de Gosson and F. Luef. A new approach to the $\star$-genvalue equation, Lett. Math. Phys. 85 (2008), 173-183

[18] M. de Gosson and F. Luef. Spectral and Regularity properties of a Pseudo-Differential Calculus Related to Landau Quantization, Journal of Pseudo-Differential Operators and Applications, 1(1) (2010), 3-34

[19] L. D. Landau and E. M. Lifshitz. Quantum Mechanics: Nonrelativistic Theory, Pergamon Press (1997)

[20] H. W. Lee. Theory and application of the quantum phase-space distribution functions, Phys. Rep. 259 (1995) 147-211

[21] J. Leray. Lagrangian Analysis and Quantum Mechanics, a mathematical structure related to asymptotic expansions and the Maslov index, MIT Press, Cambridge, Mass. (1981)

[22] C.R. de Oliveira, Intermediate Spectral Theory and Quantum Dynamics (Basel, Birkhäuser 2009).

[23] L.L. Salcedo, Statistical consistency of quantum-classical hybrids, Phys.Rev. A85 (2012) 022127.

[24] M. A. Shubin Pseudodifferential Operators and Spectral Theory, Springer-Verlag (1987) [original Russian edition in Nauka, Moskva (1978)]

[25] M. W. Wong. Weyl Transforms. Springer, 1998

\section{Author's addresses:}

- João Nuno Prata and Nuno Costa Dias: Departamento de Matemática. Universidade Lusófona de Humanidades e Tecnologias. Av. Campo Grande, 376, 1749-024 Lisboa, Portugal and Grupo de Física Matemática, Universidade de Lisboa, Av. Prof. Gama Pinto 2, 1649-003 Lisboa, Portugal

- Maurice de Gosson: Universität Wien, Fakultät für MathematikNuHAG, Nordbergstrasse 15, 1090 Vienna, Austria

E-mail address: ncdias@meo.pt

E-mail address: maurice.de.gosson@univie.ac.at

E-mail address: joao.prata@mail.telepac.pt 